\title{
Papel da via subcutânea nos Cuidados Paliativos domiciliários
}

\author{
FILIPA ALMADA LOBO*
}

\begin{abstract}
RESUMO
Enquadramento: Cerca de 60\% a 70\% dos doentes em cuidados paliativos ficam, no decurso da doença, incapazes de utilizar a via oral (VO). Uma vez que os cuidados paliativos devem ser, idealmente, prestados no domicilio, o método de aplicação mais cómoda e simples deve ser providenciado.

A via subcutânea (SC), relativamente à endovenosa, é de mais fácil utilização no domićlio: não necessitando de profissionais de saúde para a sua administração,

é menos dolorosa e pode ser utilizada durante períodos de tempo mais longos.
\end{abstract}

Descrição do Caso: Mulher de 45 anos de idade, foi funcionária da Administração Pública até Marco de 2005; Graffar de nível IV e APGAR Familiar de 9. Morou com 0 marido até essa data, e a partir dai também com a sua mãe. Não tinha filhos. Os antecedentes pessoais eram irrelevantes; ao nivel dos antecedentes familiares, verificou-se o falecimento do pai por neoplasia pancreática. Em Março de 2005, recorreu ao seu médico de família por astenia, emagrecimento, dor abdominal $e$ vómitos. Na sequência do estudo pedido, foi-lhe diagnosticada uma neoplasia do cólon sigmóide com metastização hepática. Em Maio, foi referenciada ao Instituto

Português de Oncologia (IPO) do Porto, onde, em Junho, foi submetida a uma colectomia com colostomia. Entre Junho e Outubro, por dor não controlada e/ou vómitos, teve necessidade de recorrer várias vezes ao serviço de urgência (SU), apesar da grande debilidade física de que já sofria. Em Outubro, foi referenciada à unidade de cuidados continuados do IPO. Por sua opção, encontra-se, actualmente, em casa, com assistência médica domiciliar. Por decisão conjunta, optou-se pela via SC para controlo de sintomas, sempre que, devido aos vómitos frequentes, a VO não seja possivel. Discussão: Com o caso clínico apresentado, pretende-se ilustrar situações de cuidados paliativos em que os doentes podem permanecer no domicílio com os sintomas controlados, recorrendo-se à via SC. Pretende-se também demonstrar que uma assistência domiciliar mais precoce poderia ter evitado as idas frequentes ao SU. Uma vez que a prestação de cuidados domiciliares aos doentes terminais faz parte da função de médico de família, este deve estar familiarizado com esta via de administração.

Palavras-chave: Cuidados Paliativos; Domicilio; Via Subcutânea.

\begin{abstract}
Introduction: From 60 to $70 \%$ of palliative care patients become unable, during the disease course, to take drugs using the oral route (OR). Since palliative care should ideally be home-based, the most simple and suitable application method should be provided. The subcutaneous route (SR) is easier than the endovenous route to use at home: there's no need of health professionals, it is less painful and can be used for longer periods. Case Description: 45-year-old woman, public servant until March 2005; Graffar level IV and Family APGAR score of 9; lived with her husband until that date, and since then also with her mother. No children. No relevant personal history; she had a family history of pancreas cancer in her dead father. In March 2005, she visited her family doctor for weight loss, asthenia, abdominal pain and vomiting. After the investigation, she had been diagnosed sigmoid colon cancer with hepatic metastases. In May, she was referred to Portuguese Oncology Institute (POI) of Porto, where she was performed a colectomy and colostomy. Between June and October, due to uncontrolled pain and/or vomiting, she seeked the emergency service (ES), despite her physical disabilities. In October, she went to the continuous care unit of POI. By her choice, she is currently at home with a health assistant. Whenever OR can not be applied due to frequent vomiting, SC was choosen to achieve control of symptoms.

Discussion: Based on this case report, the author highlights situations of palliative care in which patients can stay at home with controlled symptoms, using SR, and shows that an earlier home-based health assistant could have avoided the frequent patient visits to ES. Since one of the family doctor's functions is to deliver home health care to terminal patients, he or she must be familiar with this drug administration route.
\end{abstract}

Key-words: Palliative Care; Home Care; Subcutaneous Route.

\section{INTRODUCÃO}

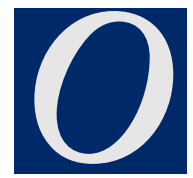

s cuidados paliativos são cuidados prestados aos doentes junto dos quais já não é possível

*Médica de Família, CS de S. Mamede de Infesta Ex-Interna Complementar de Medicina Geral e Familiar, USF Horizonte - CS de Matosinhos actuar curativamente, e que visam, mais do que proporcionar uma «boa morte», ajudar o doente a viver melhor a sua doença, através do alivio do sofrimento psicológico, físico, espiritual e social, quer do doente, quer da sua família. A Medicina Familiar, vocacionada para a prestação de cuidados integrais ao indivíduo e à família, do nascimento até à mor- te, está privilegiadamente posicionada para esta tarefa. ${ }^{1}$

A qualidade de vida e o conforto dos doentes terminais podem ser melhorados através do controlo dos sintomas, do apoio emocional ao doente e à sua família, do trabalho interdisciplinar e da adaptação flexível dos cuidados aos objectivos do doente. ${ }^{2}$ Cuidar das pessoas em fim 
de vida exige dos profissionais uma abordagem holística do doente enquanto pessoa inserida numa familia e numa comunidade.

Cerca de $60 \%$ a $70 \%$ dos doentes em cuidados paliativos ficam, no decurso da doença, incapazes de utilizar a via oral, por vários motivos: incapacidade de deglutição, vómitos, oclusão intestinal, confusão/ /agitação/delírio, estado de agonia, entre outros. ${ }^{3,4}$ Muitos estudos têm demonstrado eficácia semelhante nos fármacos administrados por via subcutânea (SC) quando comparados com os recebidos por via endovenosa. ${ }^{5-8}$ Sendo a medicação por via SC uma técnica simples, mais cómoda, mais fácil de utilizar no domicílio e associada a menos efeitos secundários que a utilização da via endovenosa - não necessitando de profissionais de saúde e não alterando a autonomia do doente nas actividades de vida diária é a opção terapêutica mais adequada quando há necessidade de administração parentérica de fármacos. ${ }^{9-14}$

Idealmente, os cuidados paliativos devem ser providenciados no domicílio. Os familiares de pacientes que morreram em casa, comparados com os daqueles que morreram institucionalizados, reportaram maior satisfação, menores preocupações com os cuidados e menos necessidades não atendidas. ${ }^{15}$ No entanto, apenas cerca de um terço concretiza o seu desejo. Inúmeros estudos demonstraram que, na grande maioria dos casos, os cuidados em fim de vida são assegurados no domicílio, por médicos de familia e profissionais de enfermagem. ${ }^{16}$

Com este caso clínico pretende-se demonstrar a importância que a via SC pode ter na prestação de cuidados adequados a doentes terminais no domicílio.

\section{DESCRIÇ̃̃O dO CASO}

\section{Identificação}

I. M. G. F.: sexo feminino, 45 anos, casada, natural do Porto e aí residente. Tem como habilitações literárias um curso técnico-profissional, tendo exercido a profissão de funcionária administrativa até Março de 2005.

\section{Antecedentes Fisiológicos}

Menarca aos 13 anos, ciclos regulares e 0 gesta.

\section{Antecedentes Patológicos}

Apendicectomia aos 20 anos e rinite alérgica.

Como antecedentes familiares relevantes há a apontar a morte do pai por carcinoma do pâncreas, a morte do avó paterno por icterícia de etiologia desconhecida e o facto de a avó materna ter sofrido de diabetes.

\section{Avaliação Familiar}

A doente pertence a uma família nu- clear que se encontra na fase I do Ciclo de Vida de Duvall. Trata-se de uma família ligada e estruturada (Modelo de Olson), altamente funcional (APGAR Familiar de 9) e com um indice de Graffar de IV.

Na Figura 1 estão representados o genograma e a psicofigura de Mitchell.

A doente encontra-se a morar com o marido, com o qual tem uma relação escassa. Não têm filhos. A relação com a mãe é excelente e com o irmão é boa.

\section{Consulta no Médico de Familia (30 de Março de 2005)}

A 30 de Março de 2005, pela primeira vez, a doente queixa-se ao seu médico de família de dor abdominal, náuseas e vómitos. Apresenta, em seis meses, um emagrecimento de 5 a $6 \mathrm{~kg}$, pele e mucosas descoradas e uma massa dolorosa palpável na fossa ilíaca esquerda. Perante este quadro clínico, o médico de família pede-lhe uma colonoscopia e uma TAC abdominopélvica.

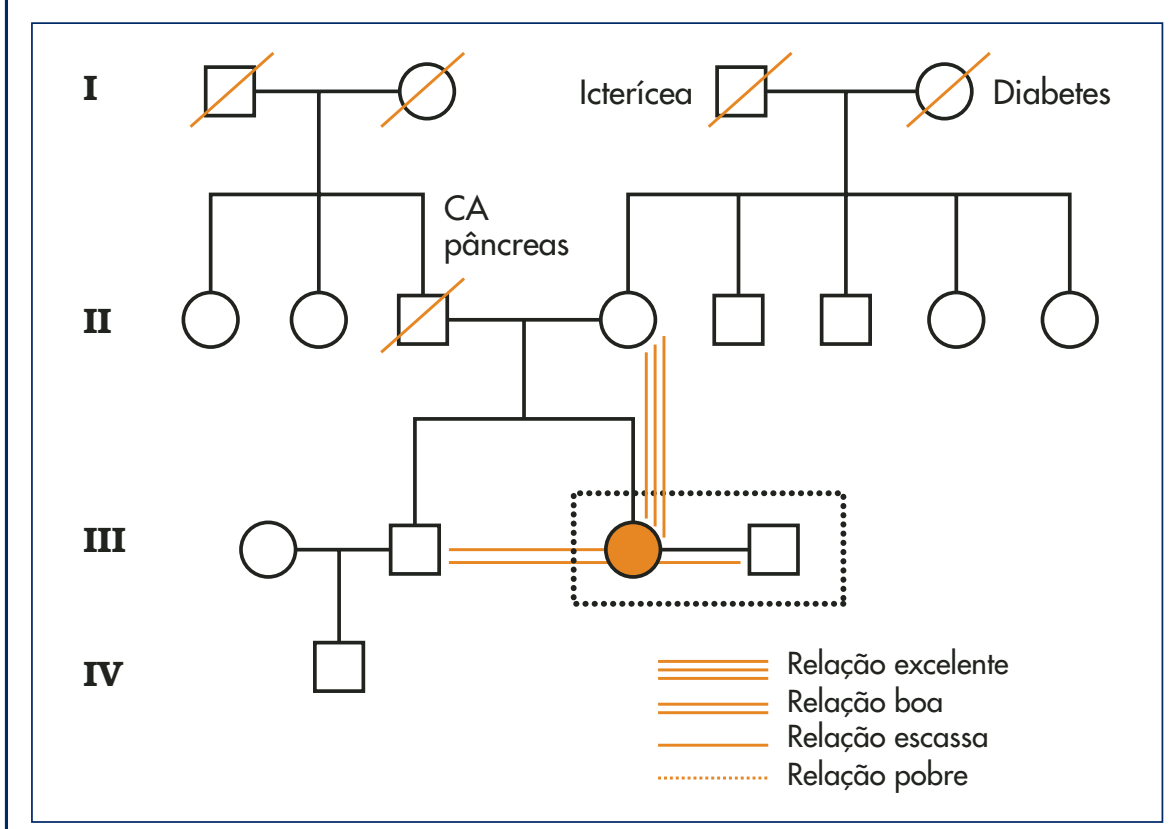

Figura 1. Representação da psicofigura de Mitchell (o círculo amarelo representa a doente). 


\section{Consulta no Médico de Familia}

\section{(15 de Abril de 2005)}

Aproximadamente 15 dias depois, a doente vai ao médico de família mostrar o resultado dos exames. Apresenta, na colonoscopia, uma lesão estenosante e vegetante a $2,5 \mathrm{~cm}$ da margem anal; a TAC abdominopélvica revela metástases hepáticas e neoformação do cólon sigmóide com extensão maior que $4 \mathrm{~cm}$. Com o diagnóstico de neoplasia do cólon sigmóide metastizada, a doente é referenciada ao Instituto Português de Oncologia (IPO) do Porto.

\section{Consulta de Grupo de Tumores Digestivos do IPO (30 de Maio de 2005)}

Cerca de um mês depois, na consulta de grupo de tumores digestivos, a doente mantém queixas de dor abdominal, náuseas, vómitos, astenia e anorexia intensas. Ao exame objectivo tem um aspecto muito emagrecido e uma massa pálpavel na fossa ilíaca esquerda; por toque rectal, verifica-se que esta se encontra a aproximadamente $8 \mathrm{~cm}$ da margem anal. É decidido programar-se uma intervenção cirúrgica.

\section{Consulta de Grupo de Tumores} Digestivos do IPO (26 de Julho de 2005)

Um mês após a cirurgia (colectomia e colostomia) mantém a sintomatologia e apresenta-se muito debilitada e com um aspecto muito emagrecido. $\mathrm{O}$ exame anátomo-patológico revela um adenocarcinoma moderadamente diferenciado que invade toda a estrutura cólica e tecido adiposo. Perante um estadio pós-operatório T4N2M1, programa-se a realização de quimioterapia paliativa.

Nas Figuras 2 a 4 representa-se a evolução sintomática da doente ao longo do tempo. Os sintomas foram quantificados utilizando a Edmon- ton Symptom Assessment Scale. ${ }^{17}$

A 27 de Julho, a doente vai à consulta de grupo da dor do IPO. Apresenta dor e náuseas de intensidade moderada a grave e fica medicada com um analgésico e um opióide (de- grau 2 da escada analgésica da OMS) e com metoclopramida. Entre 29 de Julho e 7 de Agosto, por agravamento da sintomatologia, recorre várias vezes ao serviço de urgência (SU) do IPO, não lhe tendo sido alte-



Figura 2. Representação esquemática da evolução dos sintomas entre 27 de Julho e 10 de Agosto.



Figura 3. Representação esquemática da evolução dos sintomas entre 27 de Agosto e 10 de Setembro. 

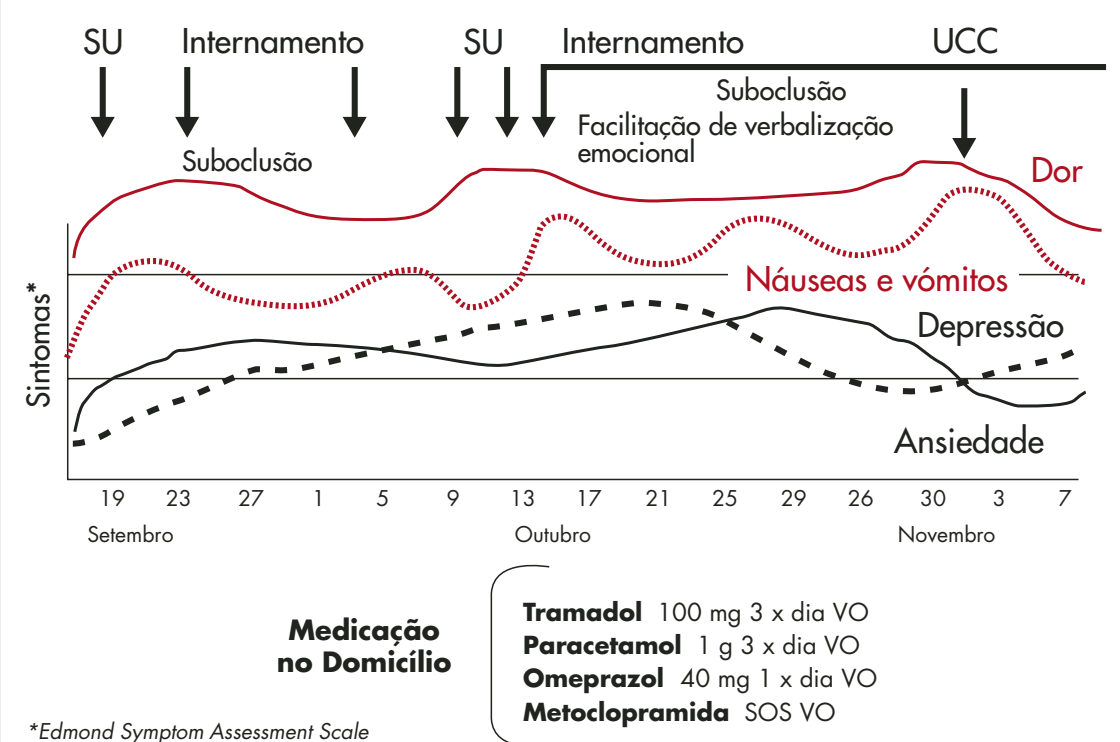

*Edmond Symptom Assessment Scale

Figura 4. Representação esquemática da evolução dos sintomas de 19 de Setembro até à referênciação para a Unidade de Cuidados Continuados.

Escala visual analógica para a dor

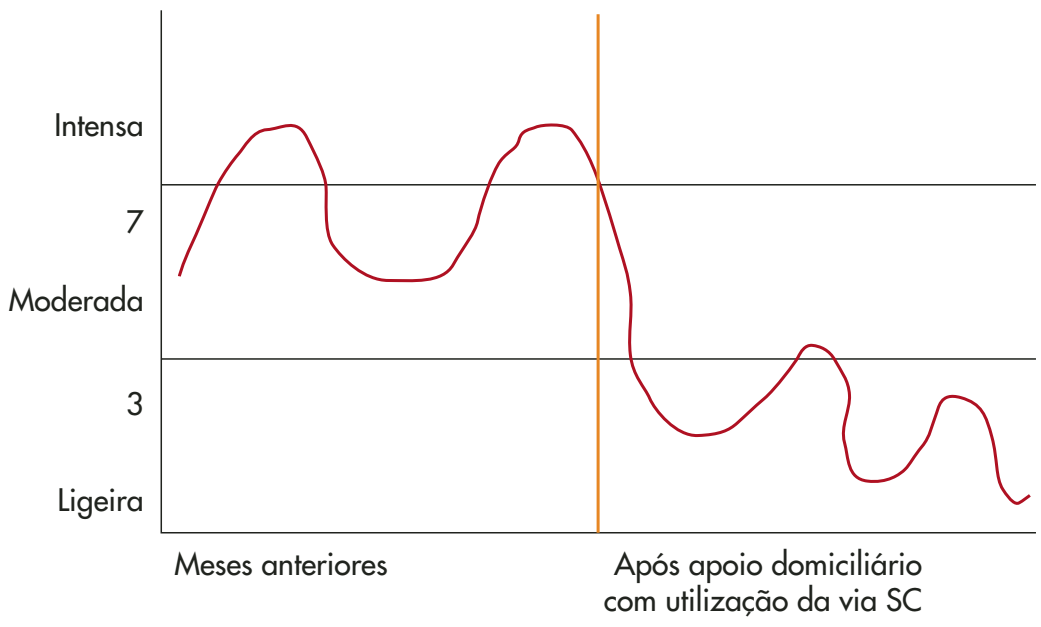

Figura 5. Esquematização da dor abdominal apresentada pela doente, representada pela Escala Visual Analógica.

rada a medicação no ambulatório. Da última vez que recorre ao SU, é internada por suboclusão intestinal (Figura 2).

A 27 de Agosto, a doente recorre novamente ao SU, sendo novamente internada por um quadro de su- boclusão intestinal. A 4 de Setembro tem alta, mantendo a medicação para a dor (um analgésico e um opióide moderado). Dois dias depois, recorre novamente ao SU por agravamento sintomático. Devido ao aumento progressivo da ansiedade, a doente é referenciada à consulta de psicologia, onde são efectuadas intervenções comportamentais e de relaxamento (Figura 3).

Como se esquematiza na Figura 4, entre 19 de Setembro e 13 de Outubro, por sintomas não controlados, recorre três vezes ao SU e fica duas vezes internada com um quadro de suboclusão. Pelos mesmos motivos, é internada de 17 de Outubro a 15 de Novembro. Associando-se aos sintomas de ansiedade, os sintomas depressivos vão-se tornando cada vez mais evidentes. Neste sentido, são utilizadas pela psicóloga várias técnicas de facilitação da verbalização emocional. Durante este internamento, é solicitada, pela primeira vez, a ajuda da Unidade de Cuidados Continuados (UCC) do IPO. A partir desta altura, verifica-se uma diminuição progressiva da gravidade dos sintomas, passando a doente a ser seguida pela equipa da UCC. À data da alta, refere uma dor ligeira (0-3 na Escala Visual Analógica) e não apresenta náuseas ou vómitos e verbaliza medo/angústia de morrer. No Modelo de Kubler-Ross, adaptado à doença em fase terminal, a doente encontra-se na fase 4, ou seja, de depressão. Mantém um aspecto emagrecido e, na Escala de Karnofsky, ${ }^{18,}{ }^{19}$ regista-se uma quantificação de $70 \%$, significando que necessita de cuidados para si, sendo incapaz de continuar a sua actividade normal. Por decisão conjunta, inicia-se, no domicílio, a administração de fármacos por via SC sempre que a via oral está restringida devido às náuseas ou aos vómitos. Para o domicílio, são prescritos os medicamentos de via SC para serem administrados em SOS, e em função dos sintomas, pela doente ou por algum familiar (Quadro I).

Desde a altura em que foi diag- 
QUADRO I

\section{MEDICACÃO PRESCRITA PARA O DOMIĆ́LIO, PARA SER ADMINISTRADA, POR VIA SC, EM SOS}

\begin{tabular}{|l|l|l|l|}
\hline Quando - em caso de: & Fármaco & Acção & Frequência \\
\hline dor contínua & morfina $10 \mathrm{mg} \mathrm{SC}$ & analgésico & até $1 / 1$ hora \\
\hline cólica abdominal & butilescopolamina $20 \mathrm{mg} \mathrm{SC}$ & anticolinérgico & até $6 / 6$ horas \\
\hline náuseas/vómitos & metoclopramida $10 \mathrm{mg} \mathrm{SC}$ & antiemético & até $4 / 4$ horas \\
\hline
\end{tabular}

Legenda - SC: subcutânea

nosticado o cancro já metastizado a doente manteve uma dor abdominal intensa a moderada; várias vezes teve necessidade de acorrer ao SU e de ser internada. Depois da referenciação à UCC verifica-se uma diminuição progressiva da dor, que se torna ligeira a moderada (Figura 5).

\section{DIsCussão}

Apesar da grande debilidade em que a doente já se encontrava, esta tem necessidade de recorrer nove vezes ao SU, em três meses e meio, por dor não controlada, moderada a intensa, e/ou vómitos. Durante este período, é internada cinco vezes por quadros de suboclusão intestinal. Durante o pouco tempo de permanência no domicílio, é medicada com opióides do degrau 2 da escada analgésica da dor da OMS, apesar de apresentar uma dor persistentemente moderada a intensa.

Em tratamento paliativo, quatro meses após o diagnóstico, a doente apresenta como principais problemas a dor abdominal e os quadros de suboclusão/oclusão intestinal. Perante um quadro de oclusão intestinal, pode considerar-se um adequado controlo sintomático se se verificarem: ${ }^{20}$

- controlo da dor contínua e em cólica (nivel da escala visual analógica menor que 3);
- ingestão, bem tolerada, de liquidos fraccionados;

- menos de 1 vómito a cada 48 horas;

- ausência de náuseas.

Foram raros os dias em que a doente não teve vómitos, tendo apresentado, como sintomas constantes, náuseas e dor. Tratava-se de uma dor persistente com picos irruptivos. Perante este tipo de queixas, teria sido importante, para além da medicação de base, a instituição de medicação para a dor irruptiva. $\mathrm{O}$ que obstou à medicação para os picos de dor foi a frequente intolerância oral, que a impedia de usar esta via.

Os cuidados paliativos surgiram como reacção ao tratamento inadequado dos doentes com doenças crónicas avançadas que não têm a possibilidade de cura ou de tratamento específico. A ideia fundamental é que estes doentes devem ser objecto de tanto interesse e atenção como os que se encontram em qualquer outra fase da doença porque, embora não se lhes possa prolongar a vida, muito se pode fazer para aliviar o seu sofrimento. $^{3}$

Os cuidados ao doente terminal baseiam-se em cinco pontos fundamentais: ${ }^{2}$

- Os cuidados devem ser integrais - tendo em conta os aspectos físicos, emocionais, sociais e espirituais - e continuados.

- O doente e a familia são a unidade a tratar, uma vez que a fa- mília é o núcleo fundamental de apoio ao doente, especialmente no que respeita aos cuidados domiciliários.

- A promoção da autonomia e da dignidade do doente tem que ser tomada em consideração nas decisões terapêuticas.

- Deve desenvolver-se uma atitude reabilitadora activa, evitando-se expressões como "não há mais nada a fazer".

- Deve ser dada importância ao ambiente, uma vez que uma atmosfera de respeito, conforto, suporte e comunicação influência de maneira decisiva o controlo dos sintomas.

Os pacientes com doença em estádio avançado, muitas vezes debilitados fisicamente e com alterações cognitivas, ficam impossibilitados de utilizar a via oral. Nestes doentes muitas vezes com pouca massa muscular devido a caquexia, e com veias quase sempre esclerosadas é muito útil a utilização da via SC. No Quadro II descrevem-se as principais circunstâncias em que é indicada a utilização da via SC e no Quadro III as principais vantagens de se recorrer a esta via.

Existem diversos estudos publicados que evidenciam a preferência dos doentes terminais em permanecer no domicílio durante a assistência à doença e em morrer em casa. Se o doente terminal morrer em casa, várias são as vantagens para todos os elementos envolvidos. O doente, no seu ambiente, consegue escolher as companhias e as actividades, expressar mais facilmente os seus sentimentos e sentir a segurança de poder morrer em casa, junto dos seus familiares; tais circunstâncias contribuem consideravelmente para a sua qualidade de vida e conforto. $\mathrm{O}$ facto de o doente estar no domicílio é também vantajoso 


\section{QUADRO ||}

\section{CASOS EM QUE É INDICADA A UTILIZAÇ̃̃O DA VIA SUBCUTÂNEA EM CUIDADOS PALIATIVOS}

\section{INDICAÇÕES}

Doentes com intolerância à VO por vómitos persistentes ou quadros de oclusão intestinal

Doentes com incapacidade de deglutição (lesões da boca, da faringe, do esófago, ou da laringe)

Doentes com má absorção intestinal

Doentes com confusão/agitação/delírio

Doentes em estado de debilidade grave/estado de agonia

Doentes sem resposta terapêutica aos fármacos tomados por VO

Doentes com necessidade de ingerir elevadas doses de medicação

Legenda - V0: via oral

para os seus familiares, uma vez que estes ficam com a possibilidade de participar nos cuidados ao doente $\mathrm{e}$ sentem menos angústia, e maior confiança e controlo. Finalmente, também é vantajoso para os profissionais de saúde, na medida em que se promove a satisfação profissional e a ligação ao doente, em que se possibilita a avaliação do grau de adaptação e funcionalidade do doente no domicílio, e em que se aumenta o conhecimento da família e de outros cuidadores informais. ${ }^{16}$

Uma revisão sistemática de 2006 procurou identificar os factores que podem influenciar a escolha dos pacientes terminais em permanecer em casa, com prestação de cuidados até à morte. Foram encontrados seis factores com uma forte associação a tal desejo: o mau estado funcional do doente, a preferência pessoal, a existência de cuidados domiciliários e sua frequência e qualidade, a coabitação com familiares e a existência de um bom suporte familiar. Deste modo, os factores podem ser divididos em três grupos, relacionados com o indivíduo, com a doença e com o ambiente. ${ }^{16}$

Este caso representa a situação de uma doente em cuidados paliativos com suboclusões intestinais recidivantes e dor não controlada; poderia ter ficado no domicilio se as três condicionantes fossem cumpridas:

- preferência da doente: a doente manifestou, desde sempre, o desejo de permanecer na sua casa, junto dos seus familiares, sobretudo junto da mãe.

- apoio familiar: a doente tinha um apoio incondicional da mãe; desde que a filha ficou debilitada, esta passou a morar com ela e com o genro;

- controlo dos sintomas: se a doente tivesse apoio domiciliário e pudesse recorrer à via SC sempre que a via oral estivesse restringida - o que nesta doente era o mais habitual.

A assistência domiciliária mais precoce, com a utilização da via SC, poderia ter evitado as idas frequentes da doente ao SU e os internamentos sucessivos. A partir do momento em que foi instituída a via SC no domicílio, assistiu-se a uma diminuição progressiva da dor e a uma menor necessidade de recorrer ao IPO, o que, dada a grande debilidade em que a doente se encontrava, foi muito importante. Para esta diminuição contribuiu também a grande acessibilidade própria da UCC, na medida em que permite que os doentes possam contactar telefo- nicamente os elementos da equipa, 24 horas por dia. A possibilidade de ter alguém para a aconselhar, ouvir e acompanhar levou a uma diminuição da ansiedade e à sua segurança e da sua família.

\section{CONCLUSÃO}

Para que os nossos doentes terminais possam ficar em casa até aos últimos dias das suas vidas devem ser antecipados cenários, deve ser promovido o ensino da família e de outros cuidadores informais, deve ser providenciado o apoio domiciliário do médico de família - enquadrado numa equipa assistencial com disponibilidade e acessibilidade - e deve ser estabelecida uma cooperação estreita com a Unidade de Cuidados Paliativos.

Fazendo parte das funções do médico de família, cuidar dos doentes terminais no domicílio é respeitar o seu desejo e significa adequar a resposta médica às suas necessidades. A utilização da via SC facilita estes aspectos; daí a importância da familiarização do médico de família, do doente e dos seus cuidadores com esta via de administração.

\section{REFERÊNCIAS BIBLIOGRÁFICAS}

1. WONCA. A definição Europeia de Medicina Geral e Familiar. Lisboa: APMCG; 2002.

2. SECPAL. Guia de Cuidados Paliativos. 2003.Disponivel em: URL: http:// www.secpal.com/guiacp/ [acedido em 20/06/2006].

3. Gonçalves F. Os últimos dias de vida. Arquivos de Medicina 1997; 11 (4): 229233.

4. Ripamonti C, Zecca E, De Conno F. Pharmacological treatment of cancer pain: alternative routes of opioid administration. Tumori 1998 May-Jun; 84 (3): 289-300.

5. Breda M, Bianchi M, Ripamonti C, 
Zecca E, Ventafridda V, Panerai AE. Plasma morphine and morphine-6-glucuronide patterns in cancer patients after oral, subcutaneous, sublabial and rectal shortterm administration. Int $\mathrm{J}$ Clin Pharmacol Res 1991; 11 (2): 93-7.

6. Moulin DE, Kreeft JH, Murray-Parsons N, Bouquillon AI. Comparison of continuous subcutaneous and intravenous hydromorphone infusions for management of cancer pain. Lancet 1991 Feb 23; 337 (8739): 465-8.

7. Stuart-Harris R, Joel SP, McDonald P, Currow D, Slevin ML. The pharmacokinetics of morphine and morphine glucuronide metabolites after subcutaneous bolus injection and subcutaneous infusion of morphine. Br J Clin Pharmacol 2000 Mar; 49 (3): 207-14.

8. Penson RT, Joel SP, Roberts M, Gloyne A, Beckwith S, Slevin ML. The bioavailability and pharmacokinetics of subcutaneous, nebulized and oral morphine6-glucuronide. Br J Clin Pharmacol 2002 Apr; 53 (4): 347-54.

9. Cherny N, Ripamonti C, Pereira J, Davis C, Fallon M, McQuay H, et al. Strategies to manage the adverse effects of oral morphine: an evidence-based report. J Clin Oncol 2001 May 1; 19 (9): 2542-54.

10. Stevens RA, Ghazi SM. Routes of opioid analgesic therapy in the management of cancer pain. Cancer Control 2000 Mar-Apr; 7 (2): 132-41.

11. Barnett M. Alternative opioids to morphine in palliative care: a review of current practice and evidence. Postgrad Med J 2001 Jun; 77 (908): 371-8.

12. Bruera E, Kim HN. Cancer pain. JAMA 2003 Nov 12; 290 (18): 2476-9.

13. Cherny NI. The management of cancer pain. CA Cancer J Clin 2000 Mar-Apr; 50 (2): 70-116; quiz 117-20.

14. Hanks GW, Conno F, Cherny N, Hanna M, Kalso E, McQuay HJ, et al. Morphine and alternative opioids in cancer pain: the EAPC recommendations. $\mathrm{Br} \mathrm{J}$ Cancer 2001 Mar 2; 84 (5): 587-93.

15. Teno JM, Clarridge BR, Casey V, Welch LC, Wetle T, Shield R, et al. Family perspectives on end-of-life care at the last place of care. JAMA 2004 Jan 7; 291 (1): 88-93.

16. Gomes B, Higginson IJ. Factors influencing death at home in terminally ill patients with cancer: systematic review. BMJ 2006 Mar 4; 332 (7540): 515-21.
17. Kaasa T, Wessel J. The Edmonton Functional Assessment Tool: further development and validation for use in palliative care. J Palliat Care 2001 Spring; 17 (1): 5-11.

18. Cuadras Lacasa F, Alcaraz Benavides M, Llort Mateu M, Madriles Basagañas MS, Martín Ramírez C, Mesalles Sin M, et al. Índice de Karnofsky's para medir la calidad de vida. Rev Enferm 1998 Jan; 21 (233): 18-20.

19. Schaafsma J, Osoba D. The Karnofsky Performance Status Scale re-examined: a cross-validation with the EORTC-C30. Qual Life Res 1994 Dec; 3 (6): 413-24.

20. Thompson I. The management of nausea and vomiting in palliative care. Nurs Stand 2004 Nov 3-9; 19 (8): 46-53; quiz 54.

\section{Endereço para correspondência} Filipa Almada Lobo

Rua Roberto Ivens, 12765.1

4450-251 Matosinhos

Telefone: 914548434

E-mail: f.almadalobo@netcabo.pt

Recebido para publicação em 13/02/2007 Aceite para publicação em 11/12/2007 